Title : will be set by the publisher

Editors : will be set by the publisher

EAS Publications Series, Vol. ?, 2018

\title{
PRECISE ASTROMETRY OF VISUAL BINARIES WITH ADAPTIVE OPTICS. A WAY FOR FINDING EXOPLANETS?
}

\author{
Krzysztof Hełminiak $^{1}$ and Maciej Konacki ${ }^{1}$
}

\begin{abstract}
We present the results of our study of astrometric stability of 200-in Hale (Mt. Palomar) and 10-m Keck II (Mauna Kea) telescopes, both with Adaptive Optics (AO) facilities. A group of nearby visual binaries and multiples was observed in near infrared, relative separations and position angles measured. We have also checked the influence of some systematic effects (e.g. atmospherical refraction, varying plate scale factor) on result and precision of astrometric measurements. We conclude that in visual binaries astrometrical observations it is possible to achieve much better precision than 1 miliarcsecond [mas], which in many cases allows detection of the astrometrical signal produced by planetary-mass object.
\end{abstract}

\section{Introduction}

Astrometry is thought to be the most promising method of exoplanets detection in the future. On the contrary to radial velocities measurements (RV), astrometry is almost independent on stellar physics, e.g. phenomena like the activity or pulsations, rather than the distance to the object. Todays interferometers are able to achieve precision at the level of mili- or microarcseconds, which is good enough to attempt exoplanet research.

Nevertheless, the same level is possible to achieve in small fields by CCD imaging with adaptive optics (AO) systems. To do that, one must subtract and correct some systematical effects in order to obtain a gaussian distribution of the measurements. In such case, the precision improves like $N^{-0.5}$, where $N$ is a number of single measurements. The goal of our studies was to check if a gaussian statistic can really be achieved with two top-class telescopes with AO systems: the 200-in Hale telescope + PHARO camera (Mt. Palomar) and 10-m Keck II telescope + NIRC2 (Mauna Kea), both working in infrared (IR). With these facilities we were

\footnotetext{
${ }^{1}$ Nicolaus Copernicus Astronomical Center, Rabiańska 8, 87-100 Toruń, Poland
} 
Title : will be set by the publisher

imaging 17 objects, majority of which were M-type dwarf binaries, located closer than $20 p c$ from the Sun.

\section{Observations}

Most of the observations were made with Hale telescope and Palomar High-Angular Resolution Observer (PHARO). The camera was using three wide-bandpass filters

- K, K' and Ks - and two narrow-band, centered on Br $\gamma$ and FeII lines (Hayward et al. 2001). PHARO's imaging mode was used with 39.91 and (mostly) 25.10 mas/pix scale. With this telescope we made about 30,000 images of 12 binaries/multiples - these are: GJ 195, GJ 352, GJ 458, GJ 507, GJ 661, GJ 767, GJ 860, GJ 873, GJ 9071 and MWC 1080 - and two fields in open clusters, i.e. NGC 1039 and NGC 6871. Observations were made between October 2001 and November 2002, during 11 nights in groups of 2 to 4 . Objects were observed in dithering mode, which means small shifts of the telescope's position in order to subtract a variable IR background and reduce the influence of the chip's distortion.

Three other multiples were observed with Keck II + Near Infra-Red Camera 2 (NIRC2). These were 56 Per, GJ 300 and GJ 596. Main components were saturated, so the astrometry could be done only for double secondarie: 1 . Keck II was used 4th March 2002 in imaging mode with 9.942 and 39.686 mas/pix scale, J, K' and K-cont (narrow-band) filters, also with dithering and, additionally, field rotation.

\section{The method}

The closure of companions allows one to observe visual binaries in a smaller field, where atmospheric distortions are weakly affecting the measurements, star's image is better sampled, and the measurements errors in pixels transfer to smaller errors in arcseconds. But, instead of parallax and proper motion, one have to take into account the orbital motion. Note also that in case of a positive detection of a 3-rd body, relative astrometry does not give the information around which particular star of the system the body is orbiting. There are also some systematic effects, which, in every astrometric research, need to be corrected in order to achieve gaussian statistics (white noise) of the measurements. For checking if the white-noise-behavior is present, we used Allan variance $\left(\sigma_{A}\right)$, which for a random dispersed values shows -1 slope on a $\log -\log$ plot of $\sigma_{A}$ vs. so-called lag.

\subsection{AO correction}

Proper AO correction is a crucial factor. Our example of GJ 352 (separation $\simeq 350$ mas $)$ shows how the quality of measurements is dependent on it. For only

\footnotetext{
${ }^{1}$ Two "secondary" stars of GJ 300 are actually field stars. The same is for GJ 873 .

${ }^{2}$ For more details see Pravdo \& Shaklan (1996) or Lane \& Muterspaugh (2004).
} 
10 properly AO-corrected frames, rms of the separation $\rho$ was 4.2 mas while for 62 others (in some of them the components were not resolved), rms reached 19.8 mas.

\subsection{PHARO chip geometry}

Depending on the position of the binary on the chip, we were obtaining various values of $\rho$, what obviously reflects variations of the plate-scale around the chip. We adopted a model of distortion with a linear dependency of the plate-scale on the $x$ and $y$ coordinates of the chip, similar to the one found by Metchev \& Hillenbrand (2004). After this correction, the measurements truly followed the white-noise-behavior (Fig. 1).

We have also found a small rotation of PHARO, between August and November 2002 , by a value of $0.641 \pm 0.013 \mathrm{deg}$. This is also in agreement with results obtained by Metchev \& Hillenbrand (2004).

\subsection{Atmospheric refraction}

Precision bellow 1 mas requires atmospheric refraction to be carefully taken into account. It's zenithal distance $(z)$ dependency causes that observed separation of two stars is smaller than the true one (due to stars' different $z$ 's). In our research we used a semi-full approach, described by Hełminiak (2008), which is suitable for IR.

\section{Precision and detection limits}

Assuming $3 \sigma$ detection limit and transferring Pravdo \& Shaklan's (1996) Equation 8 (Sect. 6, p. 270), we get a relation:

$$
a M_{P}\left[A U \cdot M_{J u p}\right]=1562.5 \frac{\sigma_{\rho} M_{S}}{\pi}\left[\frac{\text { mas } \cdot M_{\odot}}{\text { mas }}\right]
$$

where $a$ is a semi-major axis of a planet, $M_{P}$ is it's mass, $\sigma_{\rho}$ is the precision of separation measurements, $M_{S}$ is the star's mass and $\pi$ is it's parallax. $3 \sigma_{\rho}$ is used instead of the astrometric signal $\Theta$. This relation was used to calculate, what kind of planets can be found in a particular system. For stars from our sample with known mass and distance, best precision achieved and it's corresponding limits are given in Table 1. The values show that with Hale and Keck II telescopes it is possible to find Jupiter- or even Saturn-mass bodies around given stars.

For several systems, observed in all epochs, we also calculated a level of a longterm astrometric precision. We fitted 2-nd order polynomials, and also obtained rms' at the level of 100 mas or sometimes better 3 .

\footnotetext{
${ }^{3}$ Measurements, achieved precisions, limits and fittings for all systems can be found at http://www.ncac.torun.pl/ xysiek/source/mgr.pdf Ch.6, pp.94-105 (in polish, uncorrected for distortion!).
} 

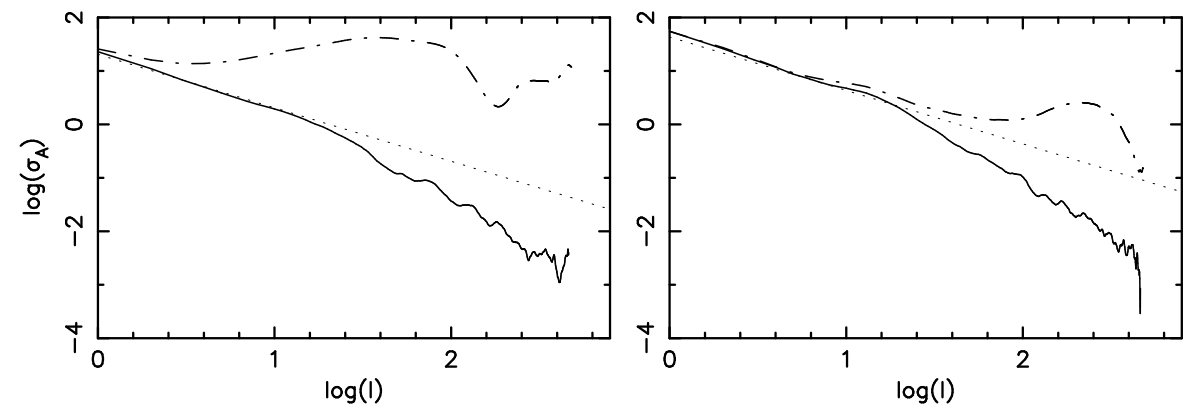

Fig. 1. Example of Allan variance vs. lag for uncorrected (dash-dotted) and corrected (solid line) measurements of separation components $x$ (left) and $y$. A small decrease is normal for finite series of measurements. Dotted line shows a -1-behavior for an ideal, infinitely long white-noise series.

Table 1. Smallest $\sigma_{\rho}$ and corresponding detection limits for researched stars.

\begin{tabular}{c|c|c|cc|cc|c}
\hline $\begin{array}{c}\text { Star } \\
\text { (GJ No.) }\end{array}$ & $\begin{array}{c}\text { Lowest } \\
\sigma \text { mas }]\end{array}$ & $\begin{array}{c}\text { Dist. } \\
{[p c]}\end{array}$ & $\begin{array}{c}\text { Mass A } \\
{\left[M_{\odot}\right]}\end{array}$ & $\begin{array}{c}\text { Limit for A } \\
{\left[A U \cdot M_{J}\right]}\end{array}$ & $\begin{array}{c}\text { Mass B } \\
{\left[M_{\odot}\right]}\end{array}$ & $\begin{array}{c}\text { Limit for B } \\
{\left[A U \cdot M_{J}\right]}\end{array}$ & Tel. \\
\hline 195 & 0.12 & 13.89 & 0.53 & 1.38 & 0.19 & 0.50 & Hale \\
352 & 1.11 & 10.53 & 0.44 & 8.04 & 0.41 & 7.49 & Hale \\
458 & 0.28 & 15.32 & 0.40 & 2.68 & 0.37 & 2.48 & Hale \\
507 & 0.33 & 13.16 & 0.46 & 3.12 & 0.37 & 2.51 & Hale \\
$569 \mathrm{~B}$ & 0.11 & 9.81 & 0.071 & 0.116 & 0.054 & 0.088 & Keck \\
661 & 0.038 & 6.32 & 0.379 & 0.16 & 0.34 & 0.15 & Hale \\
767 & 0.09 & 13.35 & 0.44 & 0.83 & 0.40 & 0.75 & Hale \\
860 & 0.048 & 4.01 & 0.34 & 0.10 & 0.27 & 0.09 & Hale \\
873 & 0.57 & 5.05 & 0.36 & 1.62 & unknown & unknown & Hale \\
9071 & 0.20 & 13.89 & 0.53 & 2.22 & 0.49 & 2.05 & Hale \\
\hline
\end{tabular}

\section{Conclusions}

Todays telescopes with AO systems allows us to perform astrometric measurements with precision well bellow 1 mas. This refers to a single-epoch observations, as well as to a long-term stability. For many cases such a precision means an ability to detect exoplanets around nearby stars.

\section{References}

Hayward, T. L. et al. 2001, PASP ,113, 105

Hełminiak, K. G. 2008, arXiv:0805.3369 2 [astro-ph]

Lane, B. F. and Muterspaugh, M. W. 2004, ApJ, 601, 1129

Metchev, S. A. and Hillenbrand, L. A. 2004, ApJ, 617, 1330

Pravdo, S. H. and Shaklan, S. B. 1996, ApJ, 465, 264 